\title{
Diffusion globale et fonctionnements locaux : géographies des scènes metal
}

Global diffusion and local functionning: the geography of Heavy Metal scenes Difusión global y funcionamiento local: la geografía de la música metal

\section{Laurent Beauguitte et Hugues Pécout}

\section{(2) OpenEdition}

\section{Journals}

Édition électronique

URL : http://journals.openedition.org/mappemonde/2017

DOI : 10.4000/mappemonde.2017

ISSN : 1769-7298

Éditeur

UMR ESPACE

Référence électronique

Laurent Beauguitte et Hugues Pécout, « Diffusion globale et fonctionnements locaux : géographies des scènes metal », Mappemonde [En ligne], 127 | 2019, mis en ligne le 01 juillet 2019, consulté le 29 mars 2020. URL : http://journals.openedition.org/mappemonde/2017 ; DOl : https://doi.org/10.4000/ mappemonde. 2017

Ce document a été généré automatiquement le 29 mars 2020. 


\title{
Diffusion globale et fonctionnements locaux : géographies des scènes metal
}

\author{
Global diffusion and local functionning: the geography of Heavy Metal scenes \\ Difusión global y funcionamiento local: la geografía de la música metal
}

Laurent Beauguitte et Hugues Pécout

Dataset : https://www.nakala.fr/data/11280/7239e0dd

\section{Introduction}

1 Le metal est un genre musical dérivé du hard rock, apparu au milieu des années 60 , et qui n'a cessé de gagner en popularité à l'échelle mondiale malgré une absence notable dans les médias grand public et un mépris critique largement répandu (Bryson, 1996; Klosterman, 2011). Genre volontiers provocateur et scandaleux, prêt à tous les excès tant stylistiques que thématiques (du satanisme à l'adulation du $\mathrm{III}^{\mathrm{e}}$ Reich), le metal est également devenu un marché économique dynamique révélé par le succès de festivals de grande ampleur dont le plus connu en France est le Hellfest (Guibert, 2012). L'existence d'une base de données collaborative très riche, le site Encyclopedia Metallum, plus connu sous le nom de metal archives, permet de retracer finement les étapes de la diffusion mondiale de ce genre musical.

2 Après avoir présenté brièvement cette base, son intérêt et ses limites, nous montrons où ce genre apparaît et comment il gagne progressivement la majeure partie de la planète. Comme tout genre musical en développement, le metal n'a eu de cesse de se spécialiser en courants de plus en plus nombreux (Martinez Garcia, 2006). Identifier les principaux courants musicaux et présenter leur géographie fait l'objet de la seconde partie. Ces approches globales, indispensables pour saisir le développement et l'emprise spatiale du phénomène, ne permettent pas de comprendre comment se 
tissent concrètement les liens entre musiciens, groupes et labels. La troisième partie, plus qualitative, s'intéresse au fonctionnement concret de deux cercles musicaux du courant le plus radical de la scène metal, à savoir le black metal national-socialiste (NSBM) ; l'objectif de cette dernière partie étant de montrer un fonctionnement simultanément très local et ouvert aux collaborations internationales.

\section{Le metal à la conquête du monde}

3 La base metal-archives, créée par deux Canadiens en 2003, est alimentée par des passionnés de metal du monde entier - plus de 85000 personnes ont contribué à cette base depuis sa création. Elle recense plus de 120000 groupes de metal pour lesquels elle fournit notamment l'origine géographique (ville ou région et pays), la chronologie de la carrière, le ou les styles musicaux et les thèmes des paroles, la composition et la discographie. Si le taux de complétude n'est pas toujours de $100 \%$ pour l'ensemble des champs, la richesse de cette base reste inégalée. De plus, les fondateurs du site ont une idée très précise, et évidemment discutable, de ce qu'est le metal: ils refusent par exemple les groupes de hard rock ( $A C / D C$, Guns'n'Roses), les groupes de nu metal (Korn, Limp Bizkit) ou de metal industriel (Rammstein, Nine Inch Nails), ces groupes jouant pourtant aux principaux festivals de metal. Le côté positif de cette vision très normative de ce qu'est le metal est l'homogénéité musicale à la base - une description plus complète de la base a été récemment publiée dans la revue Volume! (Beauguitte et Pecout, 2019a). Différents billets publiés par nos soins permettent de récolter les données (billet mis en ligne le 31 juillet 2017 : https://esprad.hypotheses.org/521) et de comprendre pourquoi et comment nous avons recodé en partie les thèmes des paroles (billet mis en ligne le 22 février 2018 : https://esprad.hypotheses.org/399) et les genres musicaux renseignés (billet mis en ligne le 12 juin 2018: https:// esprad.hypotheses.org/518).

4 Cette base a été largement utilisée par les chercheurs et chercheuses travaillant sur le sujet (Pereira, 2015) et l'un des articles les plus utiles ici est celui de Mayer et Timberlake (2014) sur les raisons favorisant la diffusion du metal. Leur hypothèse principale est que l'accès à internet a joué un rôle majeur. Leur modèle de régression multiple montre également l'importance du PIB (plus un pays est riche, plus il a de chances de voir se développer une scène metal), du niveau de démocratie et de l'ancienneté de la scène. Lorsqu'on étudie la courbe du nombre de groupes actifs par année, on se rend pourtant compte que le développement de la scène précède l'arrivée d'internet : on passe ainsi de 640 groupes en 1980 à 6000 en 1990 et plus de 20000 en 2000 (tous les chiffres de l'article sont arrondis pour faciliter la lecture), date à laquelle l'échange de musique via internet se généralise dans le grand public grâce à l'amélioration de la bande passante (Fanen, 2017).

5 Les deux cartes animées montrent l'évolution du nombre de groupes actifs par pays (figure 1) et le nombre de groupes actifs pour 10000 habitant.e.s (figure 2). L'information sur les carrières des groupes n'étant pas toujours d'une grande précision, nous avons choisi de ne représenter que les groupes dont la date de formation est connue et se situe dans la tranche chronologique indiquée. Par ailleurs, nous ne tenons pas compte ici des 650 groupes renseignés comme international. Si nous minorons le phénomène, ses logiques spatiales restent clairement lisibles ${ }^{1}$. En ce qui concerne les limites temporelles, le genre heavy metal n'existe pas en 1964, mais deux groupes 
présents dans la base (les Allemands Scorpions et les Tchèques Orient) commencent leur carrière musicale à cette date. Les effectifs étant très faibles au départ, la première tranche temporelle est de 10 ans, les suivantes de 5 ans. La date de création signale le moment où les musicien.ne.s créent le groupe, qu'il y ait ou non signature avec un label ou production discographique immédiate.

Ce média ne peut être affiché ici. Veuillez vous reporter à l'édition en ligne http://

Ce média ne peut être affiché ici. Veuillez vous reporter à l'édition en ligne http:// journals.openedition.org/mappemonde/2017

La couleur représente le nombre de groupes créés durant la période pour 10000 habitant.e.s. La discrétisation choisie est une discrétisation arithmétique en 6 classes (de 0,08 en 0,08, sauf pour la dernière classe), calculée sur la période où se trouve le taux maximum (2000-2004).

Si l'on raisonne sur le nombre de groupes, la concentration géographique reste la norme à toutes les périodes. Certes, le nombre de pays concernés ne cesse d'augmenter (10 en 1970, 44 en 1980, 90 en 1990, 120 en 2000 et plus de 140 en 2010), mais, à chaque date, les 10 premiers pays représentent au minimum $60 \%$ du nombre total de créations de groupes. Le nombre de créations de groupes est par ailleurs très fortement corrélé au nombre de groupes actifs à la période précédente (corrélation de 0,95 minimum entre une période $t$ et une période $t-1$ ). Les États où le phénomène commence à se développer tendent à rester au sommet de la hiérarchie : le trio de tête Royaume-Uni, États-Unis et Allemagne durant la décennie 1964-1974 (respectivement 12, 12 et 9 groupes) reste parmi le top 5 en 2010-2014 (première position États-Unis avec 3980 groupes, suivi de l'Allemagne -1276 groupes - le Royaume-Uni étant en cinquième position derrière le Brésil et l'Italie). Si les scènes les plus anciennes se renforcent, on note également l'apparition de nouveaux venus. Alors qu'ils ne comportaient aucun groupe de ce style durant la première décennie, le Brésil, la Russie et la France font aujourd'hui partie des dix pays où le nombre de groupes est le plus important.

Si l'on raisonne en nombre de groupes actifs pour 10000 habitant.e.s, la géographie du phénomène est différente et la hiérarchie change radicalement : les pays où le metal est le plus présent sont dans ce cas (période 2010-2014) l'Islande, la Finlande, la Suède et la Norvège. Raisonner en taux renvoie l'Allemagne à la $18 \mathrm{e}$ place et les États-Unis à la $27^{\mathrm{e}}$, derrière le Luxembourg $\left(6^{\mathrm{e}}\right)$ ou le Chili ( $7^{\mathrm{e}}$ position).

De façon assez logique, plus le nombre de groupes est important, plus on observe une forte diversité stylistique. Si l'on raisonne par nombre de groupes actifs et nombre de genres différents par État par période de cinq ans, la corrélation entre les deux indicateurs est systématiquement significative et varie entre 0,83 et 0,90 . Mais cette diversité n'est pas homogène et, des pays scandinaves foyers privilégiés du black metal à l'Amérique du Nord privilégiant le death et le thrash metal, des spécialisations nationales apparaissent clairement.

\section{De fortes spécialisations nationales}

11 Si l'on s'intéresse aux genres les plus populaires, on peut distinguer quatre grands courants devenus largement dominants depuis le milieu des années 1990: par ordre 
décroissant en nombre de groupes concernés, le death metal, le black metal, le thrash (de 15000 à 11000 groupes actifs en 2015 pour chacun de ces genres) et enfin le heavy metal (plus de 7000 groupes actifs à la même date). Sans prétendre faire œuvre de musicologue, et tout en sachant que chacun de ces sous-genres est lui-même multiple, le heavy metal reste proche du hard rock (Hein, 2004), le black metal se marque par ses atmosphères sombres (chant hurlé généralement aigu, distorsions), le thrash, très rapide et violent, aborde volontiers des thèmes sociaux et politiques quand le death, tout aussi rapide et violent, favorise les thèmes morbides et les chants graves (Berger, 2006).

Pour chacun de ces genres, des groupes précurseurs ont ouvert la voie et déclenché l'apparition de scènes locales spécialisées. La planche de cartes ci-dessous montre les résidus du chi2 ${ }^{2}$ pour les quatre genres principaux lors de la décennie 2005-2014, en ne considérant que les États dans lesquels chacun des genres est représenté par au moins un groupe (figures $\mathbf{3 a}$ et $\mathbf{3 b}$ ).

Figure 3a. Spécialisations nationales en 2005-2014 - 1
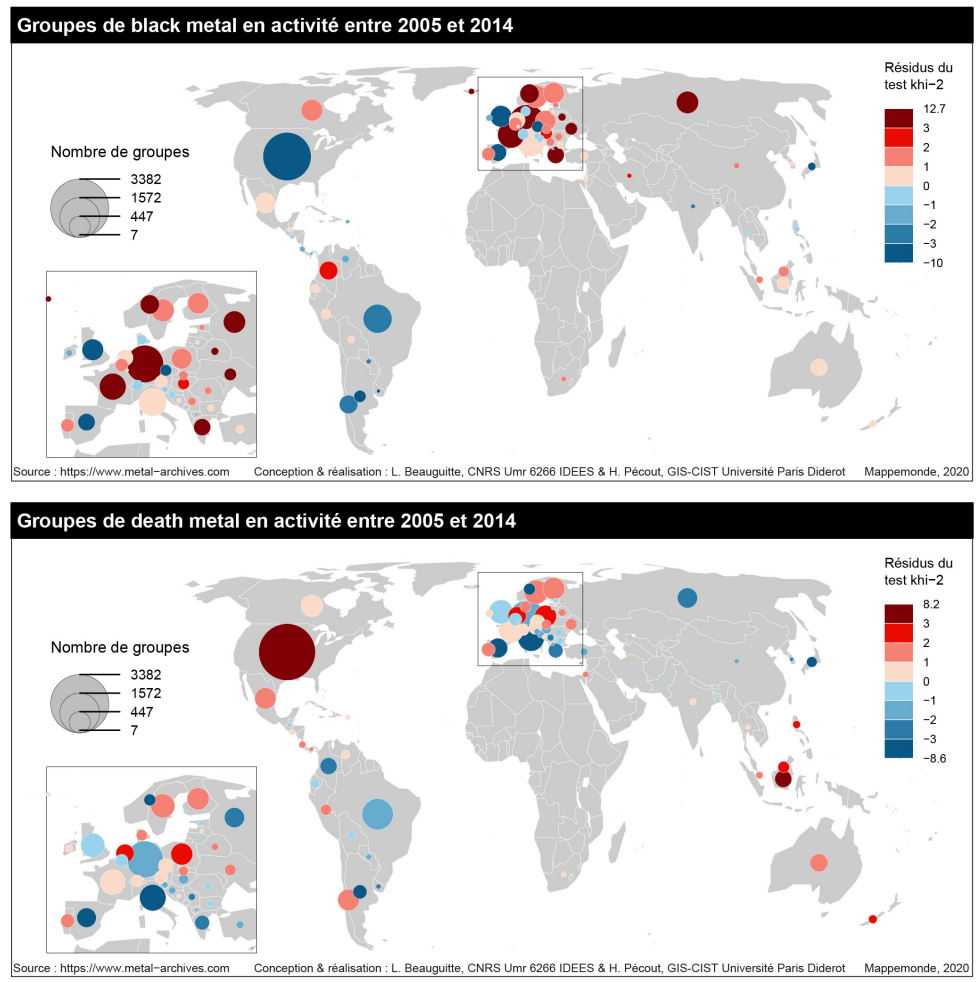
Figure 3b : Spécialisations nationales en 2005-2014 - 2
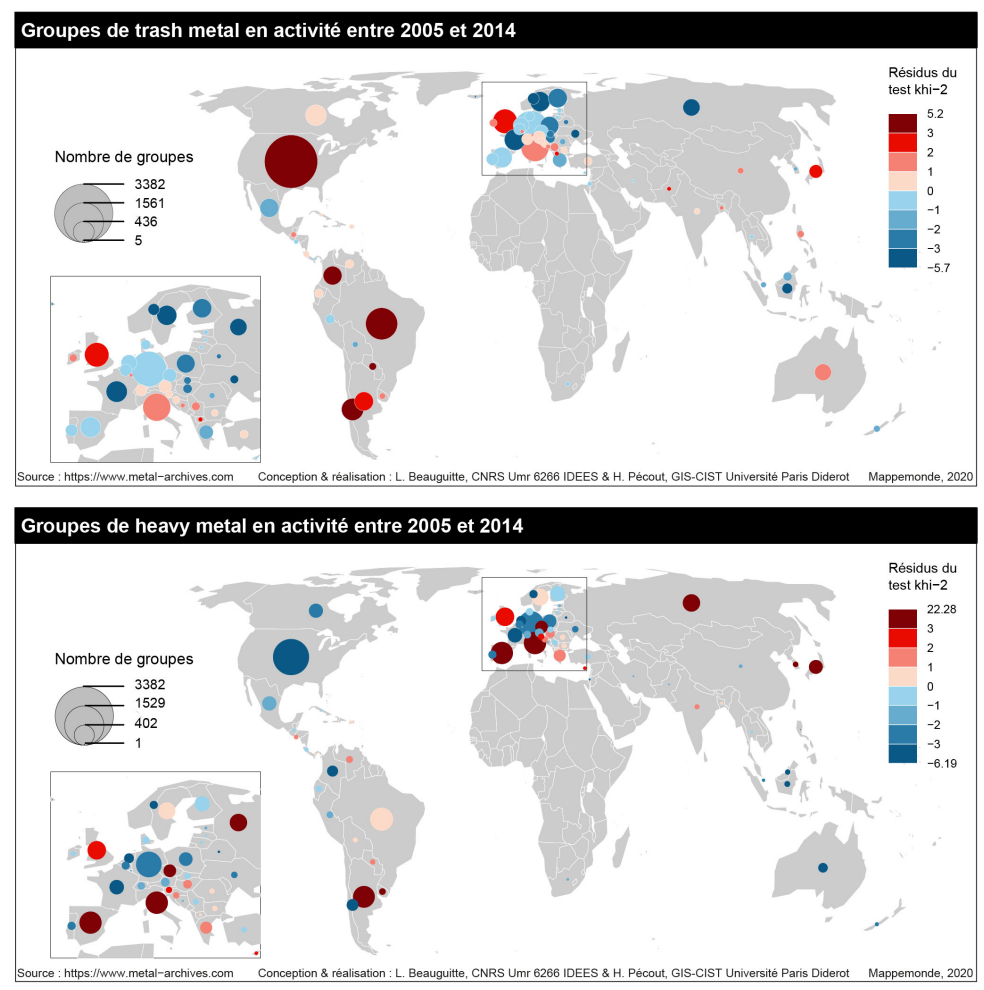

13 L'Europe scandinave, l'Europe centrale, l'Europe de l'Est ainsi que la France sont les terres d'élection par excellence du black metal et cette spécialisation s'est renforcée durant les 20 dernières années. Cette scène très active est marquée par l'importance du nombre de one-man bands (groupe formé d'une seule personne) et le refus fréquent de toute démarche commerciale (absence des réseaux sociaux numériques, autoproduction, radicalité de la musique et parfois des discours - cf. infra). États-Unis et Océanie sont davantage concernés par le death metal qui conjugue musique agressive et thèmes morbides. Le thrash, tout aussi violent musicalement, mais où les thèmes sociaux et politiques sont fréquents, est lui une spécialité américaine, des États-Unis au Chili. La structure géographique est moins nette pour le heavy metal qui concerne essentiellement deux pays d'Amérique du Sud, l'Europe du Sud, la Russie et le Japon.

Raisonner en nombre de groupes par État permet de saisir l'évolution globale du phénomène ; étudier les principaux genres permet de mettre en évidence les spécialités stylistiques. Cependant, cette approche macro ne permet pas de saisir les fonctionnements spatiaux et réticulaires des scènes metal à une échelle plus fine. Nous nous intéressons donc par la suite à deux « scènes » metal ancrées localement et à leurs principaux acteurs - l'usage des guillemets est justifié tant manquent un certain nombre d'éléments considérés comme permettant l'identification d'une scène musicale locale au sens strict (disquaire, salle de concert, lieu de sociabilité - voir Bennett et Peterson, 2004). 


\section{Clandestin, endogame et international : le cas du black metal nazi}

15 L'une des branches les plus radicales de la scène metal est celle du black metal : violente, anti-commerciale, pour laquelle choquer a toujours été un de ses objectifs. Le black metal se divise lui-même en de nombreuses catégories selon les thèmes traités et les choix musicaux. Depuis le milieu des années 90, la scène dite NSBM (national-socialist black metal) est sans doute celle qui a poussé la provocation le plus loin, entre apologie du IIIe Reich, antisémitisme exacerbé et appel au génocide (Olson, 2011). Cette scène trouve son origine en Norvège avec Vark Vikernes, unique membre du groupe très influent Burzum, qui, alors qu'il est emprisonné pour meurtre et incendies d'églises, commence à diffuser des écrits influencés par le nazisme (Taylor, 2010; Maspero \& Ribaric, 2014).

16 Si Burzum n'est pas un groupe NSBM, des centaines de groupes dans le monde entier s'approprient cette esthétique et ces thématiques. Le terme même de NSBM met quelques années à se stabiliser, les précurseurs employant des étiquettes comme Black national socialist metal (Funeral en 1996, France) ou Nazi Occult Metal (Spear of Longinus en 1995, Australie). L'une de ses premières apparitions se fait sur la compilation The Night and the Fog sortie en 1999 chez Dungeons of Darkness (États-Unis), compilation regroupant 16 groupes de 5 pays différents (dix groupes polonais, deux américains et deux français, un allemand et un autrichien). Un certain nombre de groupes s'appuient sur des cercles affinitaires ancrés localement et visant à mutualiser les coûts tout en autorisant une diffusion internationale - ce type d'organisation est courant, y compris dans le black metal non politique (l'Inner circle norvégien, Les légions noires en France, A.A.A.A. au Japon, etc.). Cette troisième partie présente deux cercles : le BrazeBirth Hall russe et le Southern Elite Circle argentin. L'objectif est de montrer que l'approche micro permet de mieux comprendre comment fonctionne une sous-culture musicale underground.

\section{Le BlazeBirth Hall russe : nationaliste, auto-centré et endogame}

Le BlazeBirth Hall, actif depuis 1994, regroupe les premiers groupes du NSBM russe, tous issus de la ville de Novomoskovsk située à 150 kilomètres au sud de Moscou. Le BlazeBirth Hall compte huit groupes de metal, dont un pour lequel aucune information n'a été trouvée (Yggdrassil). Sur les 7 groupes, 3 sont des one-man bands (Rungador, Raven Dark et Branikald). Le nombre de musiciens directement impliqués reste de ce fait relativement limité, mais leur participation à d'autres groupes, sans message politique particulier, est importante. Cette scène a été fragilisée par le meurtre d'Ulv Gegner Irminsson en 2005 et n'est plus guère active aujourd'hui; seul le groupe Rungador semble encore actif (un album sorti en 2018).

L'activité de ce type de groupes implique généralement l'animation d'un label permettant l'enregistrement et la diffusion des groupes - le label BlazeBirth Hall remplit ce rôle (46 albums sortis entre 1994 et 2018). En ce qui concerne les enregistrements, le nombre de collaborations est très faible : un split ${ }^{3}$ Nitberg/Volkoten' en 2004 et aucune collaboration discographique avec des groupes étrangers - la seule exception concerne Velimor, l'un des groupes de Ulv Gegner Irminsson, pourtant non membre du BlazeBirth Hall, avec le groupe ukrainien Kroda (un split sorti en 2006). Ce 
faible nombre de splits peut s'expliquer par deux facteurs complémentaires. Tout d'abord, la précocité de cette scène russe, regroupant une poignée de musiciens prolifiques dans une petite ville, a favorisé un développement en vase clos les premières années - on note un processus similaire en France à la même époque à Toulon avec Seigneur Voland, Funeral/Kristallnacht, Blessed in Sin, etc. Par ailleurs, si la scène NSBM est clandestine dans un grand nombre d'États (concerts clandestins, diffusion par correspondance uniquement), ce n'est pas le cas en Russie où une scène musicale d'extrême-droite existait dès avant la dislocation de l'URSS et n'a fait que se renforcer ensuite (Petra Ramet et al., 1994) - la situation a changé à la fin des années 2000, les autorités russes devenant moins complaisantes avec la scène whitepower (Dyck, 2017, p. 82-84). Pouvant sans trop d'obstacles diffuser des disques, le besoin de créer des liens avec l'étranger est sans doute moins fort.

Certains de ces groupes ayant une carrière relativement longue et un public restreint, mais fidèle, c'est notamment le cas du groupe Forest (1994-2004), les rééditions ont été fréquentes. Si l'on distingue les premières éditions et les rééditions et que l'on formalise les données sous forme de graphe biparti (liens entre groupes et labels, un lien signifiant que le groupe $x$ a sorti un disque sur le label $y$ ), on obtient les deux figures suivantes (figures $\mathbf{4 a}$ et $\mathbf{4 b}$ ). La logique générale peut être résumée ainsi ; les albums sont de préférence autoproduits ou sortent sur des labels russes (leur propre label ou le label moscovite ami Stellar Winter Records); les rééditions impliquent un nombre de labels étrangers beaucoup plus conséquents. Il faut préciser que certaines de ces rééditions sont pirates et échappent au contrôle des groupes (celles des labels The Hanged Traitor et ISO666 Releases notamment). Et, on peut le deviner au fait que la nationalité d'un des labels soit inconnue (Wolfsblood), certains de ces labels sont des structures minuscules n'ayant qu'une poignée de disques à leur actif.

Figure 4a. Liens entre groupes du BlazeBirth Hall et labels

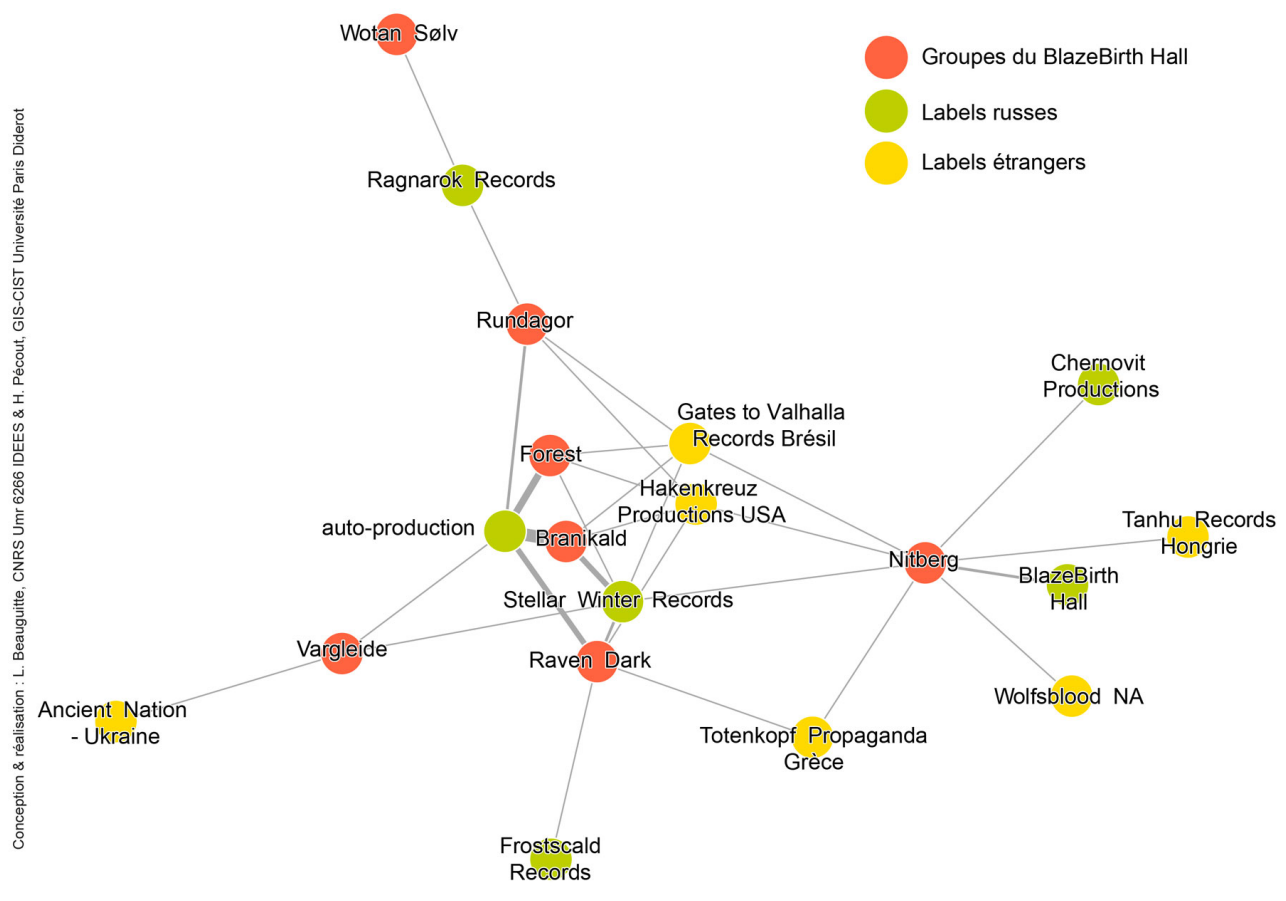


Figure 4b. Liens entre groupes du BlazeBirth Hall et labels

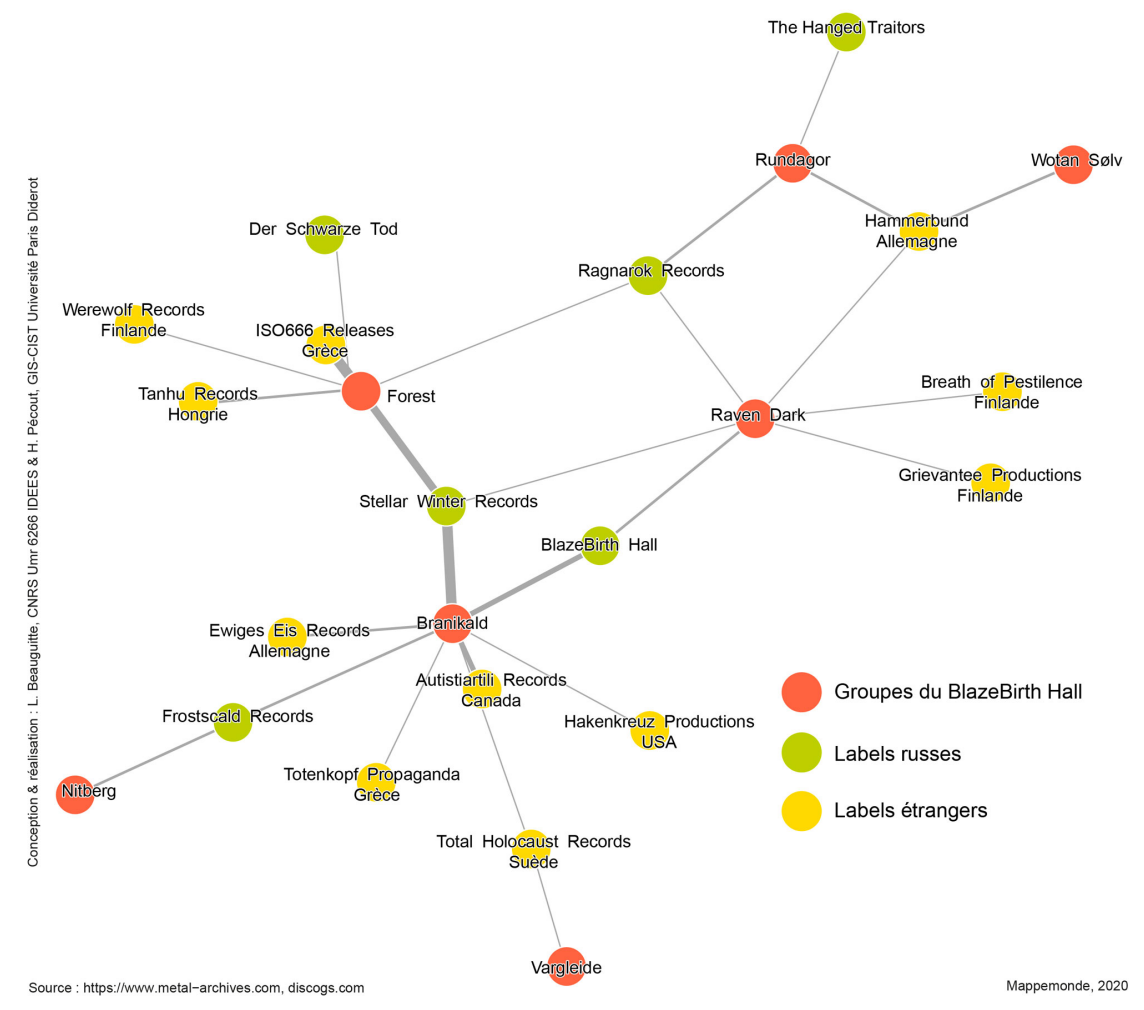

L'épaisseur des liens est fonction du nombre d'albums d'un groupe sorti par un label. L'épaisseur maximale concerne le lien Branikald - autoproduction pour les premières éditions (intensité du lien égale à 9) et le lien Branikald - Stellar Winter Records pour les rééditions (intensité du lien égale à 8).

Si la Russie et l'Europe de l'Est sont des terres de prédilection du NSBM - peu de censure jusqu'au milieu des années 2000, possibilité d'organiser des concerts, groupes ayant une longue carrière et une discographie abondante, réseaux politico-musicaux l'Amérique du Sud est une autre de ses scènes privilégiées, malgré un contexte politique souvent plus difficile.

\section{Le Southern Elite Circle : une poignée d'acteurs prolifiques et ouverts au monde}

21 En Argentine, le Southern Elite Circle basé à Buenos Aires, membre du réseau Pagan Front déjà cité, est actif depuis le début les années 2000. Le contexte légal est ici plus contraignant dans la mesure où faire l'apologie de la junte militaire d'extrême-droite (1976-1983) est un délit. Le nombre de groupes membres est légèrement plus faible que pour son équivalent russe ( 7 contre 8), mais, lorsqu'on examine la composition des groupes en question, on réalise qu'en réalité ce cercle est composé de seulement cinq personnes (figure 5) dont deux jouent un rôle prééminent. Eviigne, membre de 6 groupes du Southern Elite Circle apparaît très nettement sur le graphe de gauche. Narok, chanteur et musicien, membre de quatre de ces groupes est également le propriétaire du label Dark Hidden Productions sur lequel sortent la plupart des albums de ces artistes stylistiquement très proches les uns des autres. 
Figure 5. Un lien entre un musicien (en vert) et un groupe (en rouge) indique que le musicien a fait/ fait partie du groupe en question

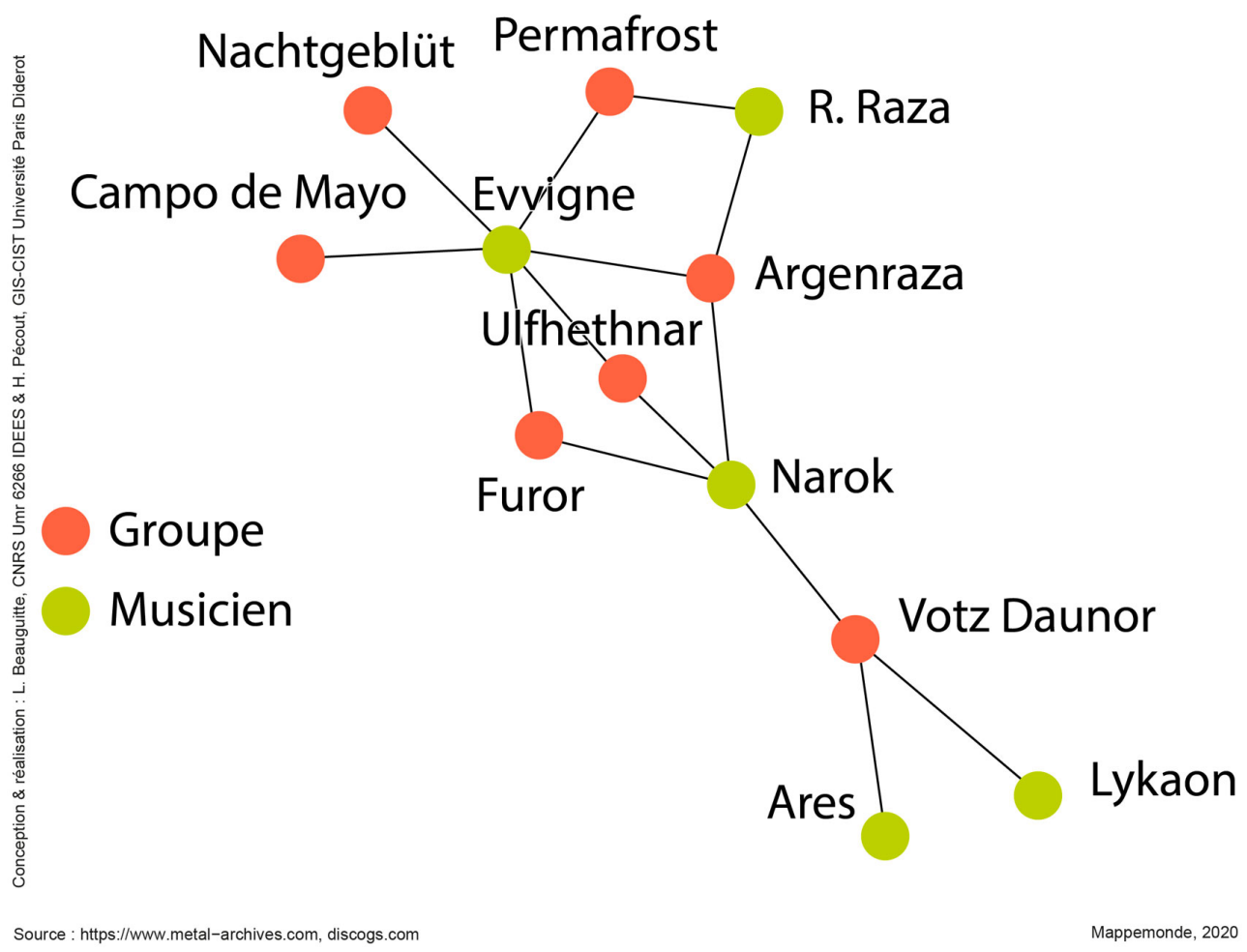

Ce micro-cercle basé à Buenos Aires et reposant sur une poignée de personnes seulement est pourtant très largement ouvert aux collaborations internationales. Des albums sont ainsi sortis sur de nombreux labels étrangers: Azermedoth Records (Mexique), Desastrious Records (États-Unis), Total War Records (Brésil), etc. Les splits révèlent également cette ouverture internationale dans la mesure où ces groupes ont enregistré avec des groupes bulgare (Aryan Art, Sofia), new-yorkais (Grafvolluh) ou français (Stormlüst).

Dans Les mondes de l'art (1982), Howard Becker évoquait deux extrêmes possibles : l'un où une seule et même personne s'occupe de l'ensemble des tâches liées à la production artistique ; l'autre où à chaque tâche correspond le rôle d'une personne très spécialisée ( situations of art making lie somewhere between the extremes of one person doing everything and every smallest activity being done by a separate person ", p. 9). Comme toutes les scènes underground (voir l'exemple des scènes anarcho-punk américaine et anglaise dans Gosling, 2004), les deux cercles présentés ici sont proches du premier cas : les mêmes personnes, impliquées dans un nombre de projets musicaux parfois conséquents, prennent en charge l'ensemble du processus de production musicale, de la composition à la commercialisation en passant par la production, l'organisation de concerts, le graphisme des albums, la distribution de labels stylistiquement et/ou idéologiquement proches, etc., etc. Pourtant, elles ne sauraient constituer des «scènes musicales locales » au sens où l'entendent Bennett et Peterson (2004, p. 1), à savoir « the contexts in which clusters of producers, musicians, and fans collectively share their common musical taste and collectively distinguish themselves from others. ». Cette possibilité de partage collectif est absente en raison de l'absence de lieux (disquaire) et d'opportunités (concert). 


\section{Conclusion}

24 Genre pluriel et dynamique, le metal est aujourd'hui présent aux quatre coins du monde et les sous-genres se comptent par dizaines. Si les États les plus développés ont les scènes les plus fournies et les plus diversifiées, le rôle des précurseurs est également notable : les États où ces scènes apparaissent restent parmi les États les plus concernés par le phénomène. Cependant de nouveaux poids lourds sont apparus sur la scène metal ces dernières décennies comme la Russie ou le Brésil.

L'examen de cercles locaux met en évidence le rôle clé joué par des artistes prolifiques (nombreux groupes et collaborations) aux activités plurielles (production et distribution, création de maisons de disques, organisation de tournées, etc.). Le caractère international de ces scènes semble en partie lié au degré d'isolement local: plus une scène est isolée - pour des raisons stylistiques ou idéologiques comme dans le cas du NSBM - plus elle tend à développer des collaborations avec des artistes et labels étrangers. Cette approche combinant analyse globale des données et examen de microscènes permet également de nuancer les chiffres bruts, notamment en ce qui concerne le black metal: les one-man bands sont nombreux et cinq groupes peuvent être l'œuvre de deux personnes seulement. Prendre en compte simultanément les trajectoires des groupes et celles des artistes est une des pistes de recherche envisagées par les auteurs pour mieux appréhender ce phénomène.

Enfin, une piste non encore explorée concerne l'étude des contributeurs de la base metal-archives. Il serait en effet intéressant d'étudier leur origine géographique et celles des groupes qu'ils renseignent: peut-on mettre en évidence des logiques locales (un contributeur commencerait par renseigner sa scène à l'échelle d'une ville puis élargirait peu à peu l'enveloppe spatiale de ses contributions) ou thématiques (choix de se concentrer sur un genre musical et un seul) ou plus vraisemblablement un mélange des deux logiques?

BIBLIOGRAPHIE

\section{Sources}

Discogs : https://www.discogs.com/

Encyclopaedia Metallum. The Metal Archives : https://www.metal-archives.com/

\section{Références}

BEAUGUITTE L., PÉCOUT H. (2019a). « Les mondes du metal d'après l'Encyclopaedia Metallum ». Volume! La revue des musiques populaires, vol. 15, $\mathrm{n}^{\circ} 2$, p. 57-69. 
BEAUGUITTE L., PÉCOUT H. (2019b). “Three NSBM circles, between local ancrage and global networks". ISMMS Conference, Nantes. En ligne : https://esprad.hypotheses.org/2334

BECKER H. S. (1982). Art Worlds. Berkeley : University of California Press.

BENNETT A., PETERSON R. A., dir. (2004). Music Scenes. Local, Translocal, and Virtual. Nashville : Vanderbilt University Press.

BERGER H. M. (2006). « Aspects du death metal ». Volume!, vol. 5, nº 2, p. 31-51. En ligne : https:// journals.openedition.org/volume/468

BRYSON B. (1996). “'Anything but heavy metal': Symbolic exclusion and musical dislikes”. American Sociological Review, vol. 61, n 5, p. 884-899.

DYCK K. (2017). Reichsrock. The International Web of White-Power and Neo-Nazi Hate Music. New Brunswick, New Jersey, Londres : Rutgers University Press, 212 p. ISBN 978-0-8135-7471-4

FANEN S. (2017). Boulevard du stream. Du mp3 à Deezer, la musique libérée. Paris : Le castor astral, 260 p. ISBN : 979-10-278-0133-6

GoSLING T. (2004). “'Not for Sale': The Underground Network of Anarcho-Punk”. In A. BENNETT ET R. A. PETERSON (dir.), Music Scenes. Local, Translocal, and Virtual, Vanderbilt University Press, p. 168-183.

GUIBERT C. (2012). « Festival Hellfest de Clisson. Les retombées économiques de la musique metal plus fortes que sa stigmatisation ». Espaces tourisme \& loisirs, n 309, p. 13-18.

HEIN F. (2004). Hard rock, heavy metal, metal. Paris : Irma, coll., « Musique et société ».

KLOSTERMAN C. (2011). Fargo rock city confessions d'un fan de heavy metal en zone rurale. Paris : Rivages, coll., « Rivages Rouge », 288 p. ISBN 978-2-7436-2281-7

MARTINEZ GARCIA S. (2006). « La production de genres : analyses depuis les périphéries du heavy metal », Volume!, vol. 5, nº 2, p. 91-113.

MASPERO D., RIBARIC M. (2014). As Wolves among Sheeps. La saga funeste du NSBM. Paris : Camion noir, 937 p. ISBN : 978-2-35779-652-2

MAYER A., TIMBERLAKE J. M. (2014). “The Fist in the Face of God. Heavy Metal Music and Decentralized Cultural Diffusion”. Sociological Perspectives, vol. 57, nº 1, p. 27-51.

OLSON B. H. (2011). "Voice of our blood: National Socialist discourses in black metal”. Popular Music History, vol. 6, n 2, p. 135-149.

PEREIRA A. B. (2015). Visualização das informações: o site Encyclopaedia Metallum: the metal archives. Curitiba: Universidade Federal do Paraná. En ligne: https://acervodigital.ufpr.br/handle/ $1884 / 41085$

PETRA RAMET S., ZAMACIKOV S., BIRD R. (1994). “The Soviet Rock Scene”. In S. Petra RAMET (dir.), Rocking the State: Rock Music and Politics in Eastern Europe and Russia, Boulder: Westview Press, p. 181-218.

TAYLOR L. W. (2010). “Nordic Nationalisms: Black Metal takes Norway's Everyday Racisms to the Extreme”. In SCOTT N.W.R., VON HELDEN I. (éd.) The metal void: First gatherings, Oxford: InterDisciplinary Press, p. 161-74. 


\section{NOTES}

1. L'ensemble des choix méthodologiques ainsi que le script $\mathrm{R}$ et le fond de carte permettant de reproduire ces traitements sont accessibles sur le blog Espaces et radicalités (https:// esprad.hypotheses.org/).

2. Le tableau correspondant n'est pas reproduit ici pour des raisons de place; le script $\mathrm{R}$ accessible en ligne permet de le générer.

3. Album partagé entre plusieurs artistes.

\section{RÉSUMÉS}

Cet article s'intéresse à l'apparition et à la diffusion à l'échelle mondiale d'un genre musical apparu à la fin des années 60, le metal. À l'aide de la base de données metal-archives qui recense plus de 120000 groupes, nous montrons à la fois la diffusion spatiale du phénomène et mettons en évidence les pays les plus touchés, tant en nombre de groupes qu'en taux de groupes pour 10 000 habitant.e.s. Ce genre étant devenu très populaire, il s'est subdivisé en une multitude de sous-genres et la deuxième partie de l'article vise à mettre en évidence les spécificités nationales. Enfin, une troisième partie plus qualitative s'intéresse au fonctionnement de deux scènes locales, mêlant affinité musicale et politique, en Argentine et en Russie. Afin d'assurer tant la reproductibilité de la recherche que l'examen d'autres questions de recherche, l'ensemble des données et des scripts $\mathrm{R}$ sont fournis en licence ouverte.

This article focuses on the emergence and worldwide distribution of a musical genre that appeared in the late 1960s: heavy metal. Using the metal-archives database with over 120,000 groups, we illustrate the spatial diffusion of the phenomenon while highlighting the countries most affected by the phenomenon, considering the number of groups and the rate of groups per 10,000 inhabitants. With the rise in popularity of this genre, a multitude of sub-genres has appeared and the second part of the article highlights national specificities. Finally, the third part focuses on how two local scenes, Argentina and Russia, function with a mixture of musical and political affinities. In order to ensure both the reproducibility of the research and the examination of other research questions, all data and R-scripts are provided under an open license.

Este artículo se interesa por la aparición y difusión mundial del metal, un género musical que surgido a finales de la década de los sesenta. A partir de una base de datos sobre este género musical, que recoge más de 120.000 grupos, se muestra la difusión espacial de este fenómeno; se destacan los países con mayor presencia numérica, o de acuerdo a tasas por cada diez mil habitantes. El metal, que es muy popular, tiene muchos subgéneros, por lo que se consagra la segunda parte a las especificidades nacionales. Finalmente, la tercera parte es más cualitativa, y se dedica a su funcionamiento en las escenas locales de Argentina y Rusia, donde se mezclan las afinidades musicales y políticas. Para asegurar la reproductibilidad de la investigación con otras consultas se proporcionan, con licencia abierta, las bases de datos y los códigos de programación para R. 
INDEX

Mots-clés : diffusion, monde, metal, musique, recherche reproductible

Palabras claves : difusión, mundo, metal, música, investigación reproducible

Keywords : diffusion, world, metal, music, reproductible research

\section{AUTEURS}

LAURENT BEAUGUITTE

CNRS, UMR Géographie-cités

HUGUES PÉCOUT

CNRS 